\title{
Freeform Fabrication of Fluidic Edible Materials
}

\author{
Humphrey Yang \\ Carnegie Mellon University, Pittsburgh, USA \\ hanliny@cs.cmu.edu \\ Kuanren Qian \\ Carnegie Mellon University, Pittsburgh, USA \\ kuanrenq@andrew.cmu.edu
}

\author{
Danli Luo \\ Carnegie Mellon University, Pittsburgh, USA \\ danlil@andrew.cmu.edu \\ Lining Yao \\ Carnegie Mellon University, Pittsburgh, USA \\ liningy@cs.cmu.edu
}
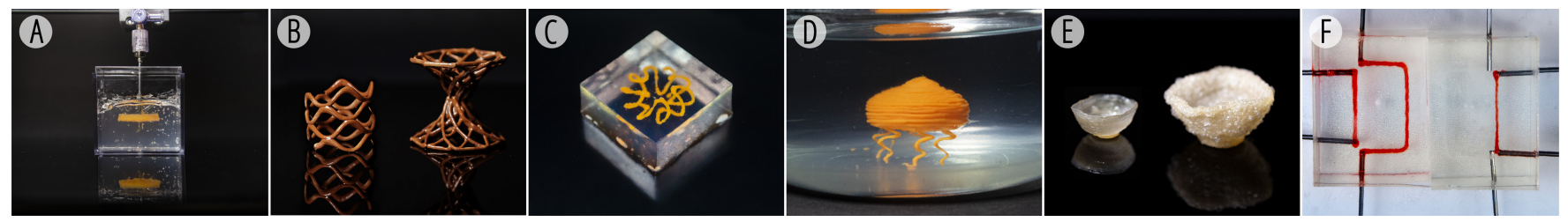

Figure 1: Fabrication method and design space overview - (A) embedded printing into a support bath, (B) 3D, self-standing chocolate structures, (C) computationally fabricated jelly and (D) alginate art, (E) freeform puffable dough before (left), and after (right) frying, and (F) dynamically controllable jelly display made of perfusable channels.

\begin{abstract}
From providing nutrition to facilitating social exchanges, food plays an essential role in our daily lives and cultures. In HCI, we are interested in using food as an interaction medium and a context of personal fabrication. Yet, the design space of available food printing methods is limited to shapes with minimal overhangs and materials that have a paste-like consistency. In this work, we seek to expand this design space by adapting support bath-assisted printing to the food context. The bath scaffolds the embedded materials and preserves shapes during the printing processes, enabling us to create freeform food with fluid-like materials. We provide users guidelines for choosing the appropriate support bath type and processing methods depending on the printing material's properties. A design tool suite and application examples, including confectionery arts, 4D printed food, and edible displays are also offered to demonstrate the enabled interaction design space.
\end{abstract}

\section{CCS CONCEPTS}

- Human-centered computing; • Human computer interaction (HCI); Interaction devices; Haptic devices.;

\section{KEYWORDS}

Food printing, edible interface, personal fabrication, computational fabrication, 3D printing

This work is licensed under a Creative Commons Attribution International 4.0 License.

CHI '21, May 08-13, 2021, Yokohama, Japan

(c) 2021 Copyright held by the owner/author(s).

ACM ISBN 978-1-4503-8096-6/21/05.

https://doi.org/10.1145/3411764.3445097
ACM Reference Format:

Humphrey Yang, Danli Luo, Kuanren Qian, and Lining Yao. 2021. Freeform Fabrication of Fluidic Edible Materials. In CHI Conference on Human Factors in Computing Systems (CHI '21), May 08-13, 2021, Yokohama, Japan. ACM, New York, NY, USA, 10 pages. https://doi.org/10.1145/3411764.3445097

\section{INTRODUCTION}

Beyond providing nutrition, food and dining also play an irreplaceable role across cultures and human activities. Recently, the HCI community has also become interested in food as an interaction platform, including using food to mediate user behaviors and sensory experiences $[3,5,13,14,18]$, display information [10, 11, 21], create shape-changing interfaces [21, 22], and enable personalization $[17,24]$. These works often use 3D printers to produce additively manufactured edible objects. However, current printing methods only navigate a limited design space in terms of either material options or printable geometries. For extrusion-based methods, the materials must have a paste-like consistency to retain their shape and cure as they are extruded, leading to prolonged printing time. The objects are often required to be flat, solid, and have minimal overhang to avoid collapses, further narrowing down the design space. On the other hand, although sintering-based food printing methods enable a larger shape space, they can only handle powdered sugar-rich ingredients [26].

To address these challenges, we adopt a bioprinting technique, embedded printing [9], which uses hydrogel baths as temporary scaffolds (Figure 1A) and apply it to various food ingredients. This modification extends the design space of food printing by including materials that are fluidic and/or require long curing time and replicating shapes that are inherently 3D and freeform with high fidelity (Figure 2). To our best knowledge, this work is the first to apply embedded printing to the fabrication of food.

We identify two challenges when we attempt to democratize embedded printing, an engineering approach previously used only by experienced material scientists and digital fabrication experts 


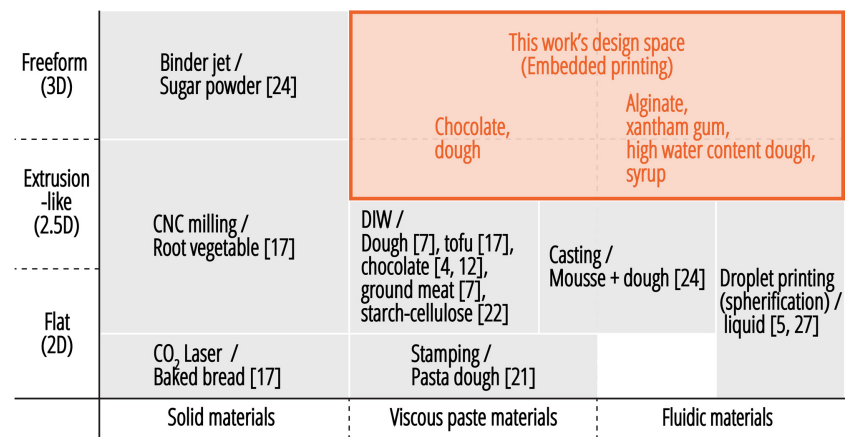

Figure 2: Current food printing design space and that enabled by this work (highlighted in orange).

before. The first challenge is how to accommodate food-specific requirements in choosing the support bath, formulating a printable ink with food ingredients, and processing or cooking the printed models. The second challenge: how do we instruct chefs and designers who have limited knowledge in material processing and additive manufacturing to customize the shape and ingredient for their food development?

In this work, we present an instructive guideline for users to formulate a compatible support bath depending on the printing materials' properties. We also develop a design tool suite to accommodate non-planar and freeform print file generation. Finally, we showcase design examples including confectionery arts (Figure 1B-D), 3D puffable food (Figure 1E), and instrumentalized jelly displays (Figure 1F) to demonstrate the design space facilitated by our technique and the associated design workflow. The contributions of this work include:

1. Guidelines for selecting and customizing support bath recipes for designers, chefs, and hobbyist makers.

2. Evaluations of embedded printing as a viable method for printing edible materials.

3. Computational design tool suite for generating freeform print files.

4. Design examples of freeform and fluidic food printing.

\section{RELATED WORK}

\subsection{Computational Food Fabrication}

Depending on the material's properties and use cases, prior works have adapted different manufacturing methods to food fabrication. For solid food materials like root vegetables, Digital Gastronomy [17] used 2.5D CNC milling machines to subtractively carve them into desired shapes. Granular materials such as sugar powder can also be built by a selective binder-jetting process [25]. As for viscous and paste-like materials, direct ink writing (DIW) has become a popular option for computational fabrication, and it has been adapted for printing a wide range of materials, including dough [7], tofu [17], chocolate [4, 12], and ground meat [7]. Compared with casting [24] or stamping [21] that require pre-manufactured molds, DIW allows users to conveniently fabricate different shapes by swapping print files, thus making it an ideal platform for personalizing food. Beyond shapes, DIW also enables users to control the texture or mouthfeel [15] and transformative behaviors [22] by modulating its structure. However, since extruded materials may take time to solidify and would deform or flow under gravity, DIW is limited to replicating shapes that are flat or resemble an extrusion. Alternatively, while $[5,27]$ uses liquid spherification to produce 3D shapes out of edible liquids, the resolution is limited to the droplets' size, and the prints are still prone to collapse under gravity. In this work, we seek to expand the toolkit of food fabrication by augmenting DIW to accommodate fluidic materials and intrinsically 3D shapes (Figure 2). Beyond the customization of food, this extension may also enable us to explore human-food interactions in broader scenarios.

\subsection{Embedded Printing in Engineering and Design}

Besides hard materials, researchers had also become interested in the additive manufacturing of soft matters. Embedded printing, in particular, is a bioprinting technique developed for printing silicone [23], collagen [9], and even living cells [2]. The printing process is identical to conventional 3D printing - building objects by depositing layers of materials. Rather than printing onto a flat platform, this technique directly prints inside a support bath that scaffolds the extruded materials. The bath is made of biocompatible or edible hydrogels with a shear-thinning property, which allows the bath to locally fluidize under the shear force of a moving extruder and become pseudo-solid when undisturbed, providing omnidirectional support for the extruded materials. The printed object can be released afterward by rinsing off the support bath or remain inside a curable bath.

In other domains, Hajash et al. had also adapted this fabrication method to produce industrial products made of resin, rubber, and concrete [6]. Although various support bath recipes, such as gelatin slurry and Carbopol, are already available in the literature, they raise specific concerns regarding the $\mathrm{pH}$, melting temperature, additives, release methods, etc. when applied to food printing. Thus, we provide guidelines for users to choose the appropriate bath type depending on the food material properties and processing methods. Similarly, we introduce several bath removal strategies adapted for food materials with different properties (e.g., brittleness, melting point).

\section{GUIDELINES}

This section provides guidelines to encourage designers, chefs, and hobbyist makers to employ embedded printing in their food preparation practice. We believe these guidelines are practical to adapt with minimum culinary science knowledge.

\subsection{Design Space}

In this work, we show five food material printing examples with different properties and support bath requirements. The support bath type and release method for each material is determined by following the guidelines shown in Figure 4. Based on the embedded printing technique, we also develop a design tool specific to the freeform path planning with non-planar nozzle movement. This further aids the design and fabrication following these guidelines, 


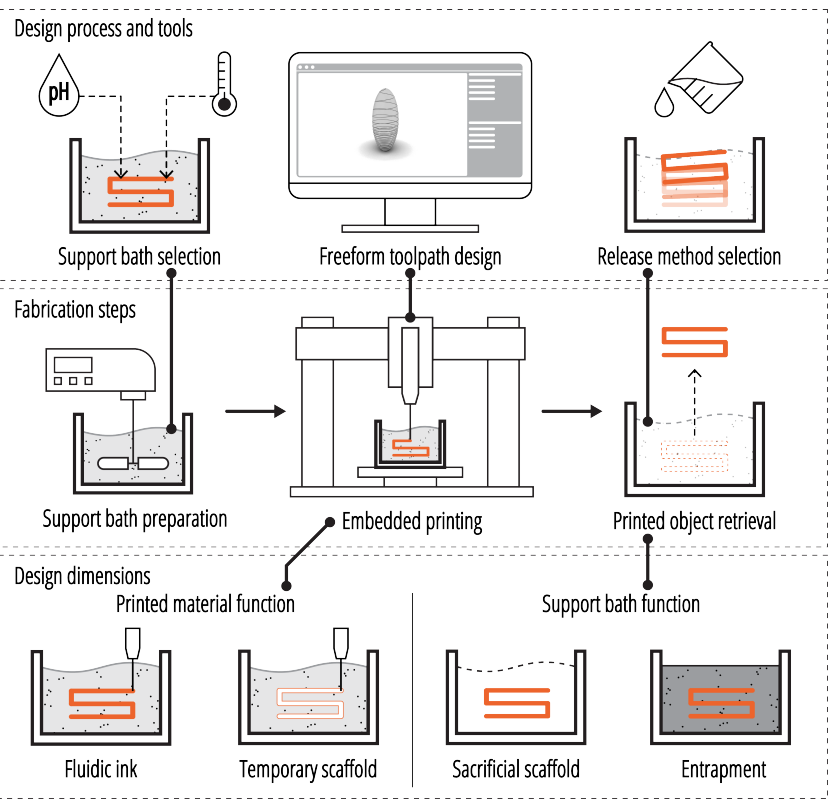

Figure 3: Freeform and fluidic food printing overview.

and we explore several design dimensions associated with freeform food printing (Figure 3, see also Application Examples section).

\subsection{Support Bath Selection}

Having perused a rich literature on how scientists prepare support baths for embedded printing, we compare and contrast two types of support baths to accommodate different food ink propertiesone made of gelatin $[9,20]$ and one made of Carbopol. Gelatin is derived from animal tissue as the main ingredient for making jelly, thus it is inherently edible. Carbopol is a polyacrylic acid powder with a chemical name of carbomer [2,6] and commonly used in cosmetics and medications as a thickener and is considered safe to use in food packaging [28].

When choosing the right support bath, the food ink's $\mathrm{pH}$ value and printing temperature are two important factors as the Carbopol and gelatin support baths react to both factors differently. Carbopol baths are stable across a wide temperature range. Their ability to retain viscosity varies with the $\mathrm{pH}$ value and the presence of ionic substances (e.g., salts). Though gelatin is compatible with food inks that have different $\mathrm{pH}$ values and is insensitive to ionic substances, the low melting point $\left(32^{\circ} \mathrm{C}\right)$ renders it incompatible with inks that require warm extrusion or heat processing. In addition, while the gelatin support bath used in the related bioprinting works is sacrificial and will be removed by heating above $32^{\circ} \mathrm{C}$, we have an option to utilize its food-derived nature and keep the bath as part of the food. In this case, an additional part of a gelatin solution is added to the gelatin bath to cure it, and the embedded liquid remains trapped inside the gelatin block.

Figure 4A shows the guidelines for choosing the appropriate support bath based on the printing ink's properties. In this guideline, the material's $\mathrm{pH}$ value and temperature requirements are the deterministic factors. Note that both the extrusion and the processing temperature (e.g., thermal-setting the dough's shape before releasing them from the bath) should be considered when determining the ink's temperature requirements. If a material is compatible with either support baths, users are advised to use the Carbopol bath for its 12.5-times-faster and more straightforward preparation process. Finally, the two support baths can already accommodate a wide range of printing materials. Still, we acknowledge that some materials (i.e., ionic inks that require $>32^{\circ} \mathrm{C}$ extrusion or thermal processing, materials that require subzero or $>100^{\circ} \mathrm{C}$ treatments) are still not compatible with the current method.

\subsection{Release Methods}

Similar to support bath selection, the print-release method also depends on the material properties of both bath and ink (Figure $4 \mathrm{~B})$. As long as it is unreactive with the ink material, the stimuli to which the support bath is sensitive to can facilitate the release process. Specifically, the gelatin bath can be dissolved by heat, and the Carbopol bath can be liquified with ionic solutions (e.g., lemon juice, bivalent salt) to make the object easier to retrieve. If these methods are incompatible with the ink material, users are advised to use cold distilled water to rinse away the supports. On the other hand, if the printed object is delicate, users can also submerge the printed object and bath as a whole into a tank of water and remove the bath by gently swirling them.

\section{METHODS}

This section details the material preparation and fabrication platform that has been adapted to embedded food printing.

Table 1: Comparison of support bath material properties.

\begin{tabular}{lll}
\hline BATH MATERIAL & Carbopol & Gelatin \\
\hline NATURE & Water-soluble gel & Granular gel slurry \\
EDIBLE & No & Yes \\
WORKING TEMPERATURE $\left({ }^{\circ} \mathrm{C}\right)$ & $0-100^{\circ} \mathrm{C}$ & $4-32^{\circ} \mathrm{C}$ \\
PH TOLERANCE & $5-8$ & Insensitive to pH changes \\
PREPARATION & 4.8 & 60 \\
TIME $($ MIN $/ 100 M L)$ & & \\
CURABLE BATH & No & Yes (by adding gelatin solution) \\
RELEASE METHOD & Acidify or dissolve with distilled water & Rinse with cold water or dissolve at $32^{\circ} \mathrm{C}$ \\
\hline
\end{tabular}




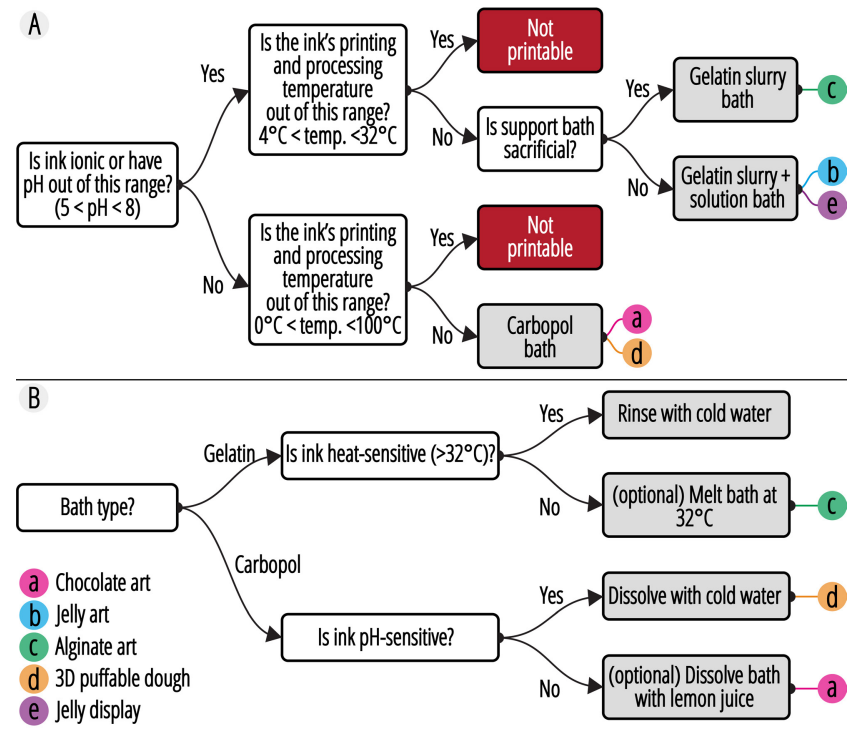

Figure 4: Guidelines for (A) selecting support bath types and (B) release methods. Support bath selection guidelines.

\subsection{Material Preparation}

All our materials are accessible and readily available from food suppliers. Specifically, gelatin, calcium chloride $\left(\mathrm{CaCl}_{2}\right)$, alginate, and xanthan gum are purchased online from Modernist Pantry. Vegetables, chocolate, syrup, starch, flour, and oil are purchased from a local grocery store.

4.1.1 Gelatin Bath. For the gelatin bath to be shear thinning like wet sand, it has to be gelled and blended into a microparticle slurry. We follow Hinton et al.'s protocol with minimal changes for making gelatin slurry baths [9]. $6.75 \mathrm{~g}$ of gelatin is first dissolved in $150 \mathrm{~mL}$ of $11 \mathrm{mM} \mathrm{CaCl}_{2}$ (the molar concentration translates to $1.22 \mathrm{~g} / \mathrm{L}$ of distilled water) solution at $60^{\circ} \mathrm{C}$ and gelled for 12 hours at $4^{\circ} \mathrm{C}$. The gelled gelatin is then blended in $350 \mathrm{~mL}$ of $11 \mathrm{mM} \mathrm{CaCl}_{2}$ solution for 2 minutes with a blender. Next, the mixture is left to sediment before the supernatant and bubbles are removed, leaving the slurry at the bottom. $350 \mathrm{~mL}$ of fresh $11 \mathrm{mM} \mathrm{CaCl}_{2}$ solution at $4^{\circ} \mathrm{C}$ is added again to resuspend the slurry. The steps from removing supernatant to adding fresh $\mathrm{CaCl}_{2}$ solution are repeated 3 to 5 times until the supernatant turns up clear, which indicates that dissolved gelatin is completely removed. Next, the slurry is centrifuged at $4,000 \mathrm{rpm}$ for 5 minutes to remove the excess $\mathrm{CaCl}_{2}$ solution. At this point, the slurry is ready for use and can be preserved for up to 7 days at $4{ }^{\circ} \mathrm{C}$. Note that batch preparation of gelatin slurry is possible at the blender's maximum capacity, but we recommend preparing as much as needed before the gelatin stales.

The curable gelatin bath is prepared by mixing the slurry with a $10 \%(\mathrm{w} / \mathrm{v})$ gelatin solution (dissolved in $100 \mathrm{mM}$, or $11.1 \mathrm{~g} / \mathrm{L}$, $\mathrm{CaCl}_{2}$ solution at $32^{\circ} \mathrm{C}$ ) at an $85: 15$ ratio prior to printing. The bath remains printable for an hour under room temperature $\left(25^{\circ} \mathrm{C}\right)$, and the finished print should be left to gel in a refrigerator for at least 4 hours. This protocol is adapted from [20].
4.1.2 Carbopol Bath. We modified the procedure in [8]. First, Carbopol powder is added to filtered water and mixed for 3 hours to create a homogeneous $5 \%(\mathrm{w} / \mathrm{v})$ stock solution. Next, the solution is neutralized with $0.416 \mathrm{~mL}$ of $10 \mathrm{M} \mathrm{NaOH}(400 \mathrm{~g} / \mathrm{L})$ solution per $100 \mathrm{~mL}$. Note that neutralization is needed for inducing the shear-thinning property of Carbopol. When stored in a sealed container, the prepared material can be preserved for up to a month under room temperature. Before printing, a 1\% Carbopol solution is diluted from the stock solution by simply adding distilled water.

4.1.3 Jelly and Alginate Art Inks. Using gelatin - a hydrophilic material - as the support bath limits the colorant options to waterinsoluble materials, or else the dye would diffuse into the bath. We use two types of inks to circumvent this issue - colored fiber-laden or syrup-based alginate inks. To prepare the fiber-laden ink, we $\operatorname{mix} 1 \%(\mathrm{w} / \mathrm{v})$ alginate solution with diced carrots at a 1:1 weight ratio and blend the mixture for 10 minutes to arrive at a consistent suspension. Here, alginate is added to bind the loose vegetable fibers. As alginate has been used widely in combination with $\mathrm{CaCl}_{2}$ to perform liquid spherification [19], we take advantage of this process to create a non-water permissive membrane as the ink is extruded into a gelatin bath. To prepare the syrup-based ink with alginate as a barrier, an off-the-shelf chocolate syrup (Hershey's) is mixed with $0.5 \%$ of alginate.

Similarly, the alginate art ink cures inside the bath before release. $1 \%(\mathrm{w} / \mathrm{v})$ alginate solution is mixed with the same weight of chopped carrot. The solution is blended for 10 minutes and strained through a 200-mesh sieve.

4.1.4 Chocolate Ink. The chocolate ink is printed in the Carbopol support bath to create curable, retrievable, and self-standing structures. We melt and stir off-the-shelf chocolate $(72 \%$ Cacao chocolate bar, Ghirardelli) at $35^{\circ} \mathrm{C}$ until smooth. During fabrication, the extruder's reservoir is also heated to $35^{\circ} \mathrm{C}$ to prevent the ink from solidifying.

4.1.5 Puffable Dough Paste. Dough with a high starch content undergoes large expansion when fried [16], and we leverage this property to create puffable objects. Starch, wheat flour, and filtered water are mixed at a 1:1:1.5 weight ratio and stir until homogeneous. After being printed into the Carbopol bath, the dough is steamcured as is at $100^{\circ} \mathrm{C}$ for 35 minutes to set its shape. Once cured, the dough is retrieved by rinsing away the Carbopol bath. From this point on, the dough can be further processed without losing its shape. The dough is allowed to dry for 24 hours before it is fried in $180^{\circ} \mathrm{C}$ vegetable oil.

4.1.6 Xanthan Gum Ink. Xanthan gum is a common thickener for making salad dressing. When injected into a gelatin bath, the ink can scaffold the printed channels while the bath is curing and be washed away later to create perfusable hollow channels for liquids to flow through [20]. To prepare the ink, xanthan gum powder is added to filtered water at a $1 \%(\mathrm{w} / \mathrm{v})$ ratio, and the solution is stirred for 12 hours to obtain a homogeneous solution. The ink is centrifuged before printing to remove trapped air bubbles. 


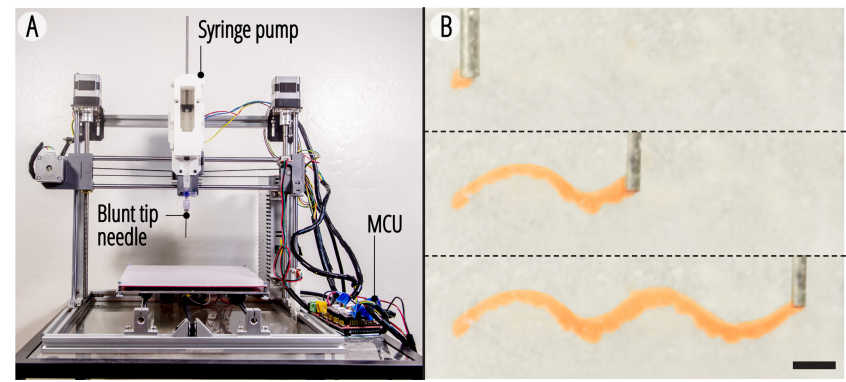

Figure 5: Fabrication system - (A) printer setup and (B) printing process (gelatin bath with alginate-carrot ink). Scale bar: $5 \mathrm{~mm}$.

\subsection{Fabrication Platform}

Two printers are used in this work: a consumer-grade printer retrofitted with a syringe pump (Figure 5A) is used to print all of the ink materials except for the chocolate ink, and a modular DIW printer (Hyrel 30M) is used for printing the chocolate ink at an elevated temperature $\left(35^{\circ} \mathrm{C}\right)$. Note that besides the heated reservoir, there is no functional difference between these printers. Both printers take conventional G-codes as input and allow non-planar movements in 3D space (Figure 5B). However, when generating print files, the print speed must be sufficiently high $(2.5 \mathrm{~mm} / \mathrm{s})$ to trigger the support baths' shear-thinning behavior (i.e., to become fluidic for smooth movements). Before pouring in support bath materials, the print container's inner surfaces are brushed with cooking oil to make printed objects and support bath easier to remove. All materials were printed with a 16-gauge blunt tip, 2-inch-long needle except for the carrot fiber ink, which was printed using a 14-gauge, 2-inch-long needle.

\section{EVALUATIONS}

This section uses two experiments to assess embedded printing's ability to handle freeform geometries and fluidic materials. Both investigations are based on widely used food inks (i.e., chocolate and dough), and the results are compared with those fabricated on the same printer but without a support bath. In the Freeform Printing Test, we show that embedded food printing allows us to create structures with more than $3 x$ larger overhangs compared to conventional printing. The Fluidic Material Printing test shows that embedded printing can accommodate fluidic materials without observable defects.

\subsection{Freeform Printing}

Though chocolate printing has been explored in engineering [12] and commercial contexts [4], it is difficult to print inherently 3D structures with available techniques and recipes. Specifically, extruded molten chocolate tends to flow due to gravity before it is cooled into solids, making it difficult to print large overhangs. In this test, we use the chocolate ink (see Material Preparation) to print the same structure with both conventional and embedded printing techniques (print speed: $5 \mathrm{~mm} / \mathrm{s}$, flow: $30.4 \mu \mathrm{L} / \mathrm{s}$ ). The structure (Figure 6A) is designed as a single-line-thick shell with an increasing slope along the vertical axis, such that when printed from the

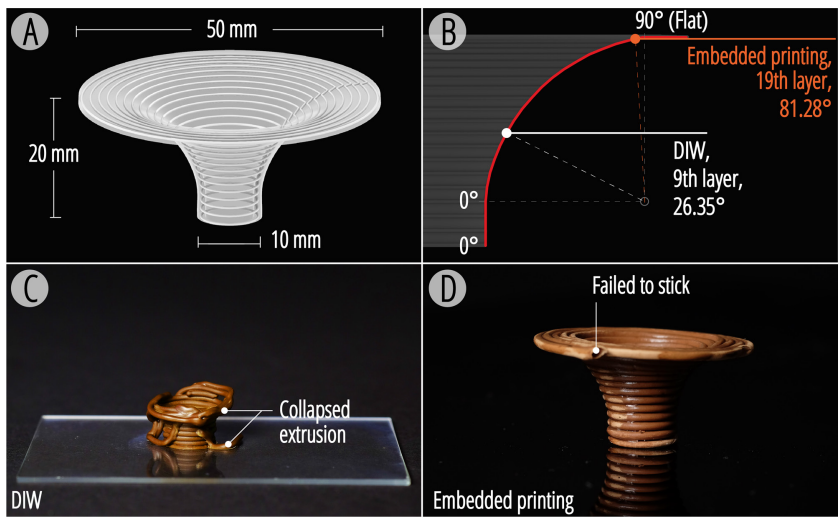

Figure 6: Freeform printing test - (A) the model used for this experiment has (B) an increasing overhang along the $z$-axis. (C) The model printed with DIW and (D) embedded printing in a Carbopol support bath.

bottom-up, each succeeding layer would have a larger overhang compared to the previous layer (Figure 6B). By comparing the layer at which the print starts to fail, we can identify the maximum overhang achievable by that printing method.

Results show that when using DIW, the print would start to fail at the 9th layer, which has a $26.35^{\circ}$ overhang (Figure 6C). By contrast, embedded printing could resume printing until the 19th layer, which is almost printing on a horizontal plane $\left(82.28^{\circ}\right.$, Figure $6 \mathrm{D})$. This improvement is due to the support bath's shear-thinning properties - as soon as the molten chocolate comes out of the nozzle and the extruder moves away, the surrounding Carbopol becomes all-directional support to prevent it from flowing as it solidifies. On the other hand, although the chocolate failed to stick at the 19th layer, we observe that the failure only occurred as the chocolate is released from the support bath and cannot sustain its weight. In a follow-up test, we found that reducing the line spacing from $1.5 \mathrm{~mm}$ to $1.25 \mathrm{~mm}$ (i.e., $83 \%$ of nozzle diameter) allowed us to produce a complete (i.e., 90 degree) overhang with good adherence between layers and without structural failure. In addition to this print experiment, Figure 11 also shows that embedded food printing inherently affords non-planar toolpaths and large bridging.

\subsection{Fluidic Material Printing}

Dough, made of flour and liquid (e.g., milk, water), is an exemplary food material that may have a wide range of properties, viscosities, and design spaces depending on the ratio between its ingredients. For instance, dough with low water content is viscous and can be easily modeled into $2.5 \mathrm{D}$ shapes for baking cookies or bread, while dough with high water content is more fluidic and deformable and is widely used for making puffable flat food like pizza. As a result of this difference in fluidity, current 3D printing techniques are mostly limited to using low-hydration dough for making foods. In this work, we use dough with different water content levels for validating fluidic material printing. Its ingredients are also standardized, accessible, and common products, making it an ideal model material of liquid foods. The same recipe was also used for producing the puffable dough example (Figure 14) in this paper. 


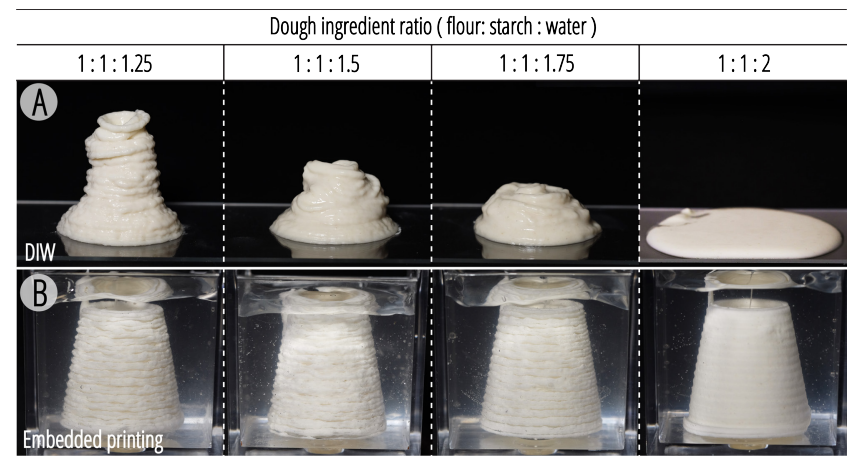

Figure 7: Fluidic material printing test showing dough with different water content levels printed with (A) DIW and (B) embedded printing in a Carbopol support bath.

In this experiment, we show that embedded printing is more capable of handling fluidic materials than DIW. We use both printing methods to fabricate a hollow frustum $(30 \mathrm{~mm}$ wide, $30 \mathrm{~mm}$ tall, 2-layer shell) with the same print file (print speed: $2.5 \mathrm{~mm} / \mathrm{s}$, flow: $4.7 \mu \mathrm{L} / \mathrm{s}$ ) and vary the dough's hydration ratio between each test. Results show that the samples printed with DIW tend to collapse across all hydration rates due to gravity (Figure 7A). The degree of collapse also depends on the dough's water content - the more hydrated the dough is, the more flattened and spread-out the resulting object is. In comparison, there was no observable difference between the frustums printing in support baths - all frustums were able to retain their shape regardless of the material (Figure 7B).

\section{DESIGN TOOL}

\subsection{Implementation}

We implement a design tool comprising a suite of toolpath design functions in Rhinoceros 3D and Grasshopper to allow users to convert geometries into freeform fabrication files. The interface (Figure 8) contains two windows - a model viewport and a graphical scripting interface. In the scripting interface, users can drag and connect the toolpath design functions (Figure 9A, B left-hand side) and assign parameters to customize print files. The generated toolpath is rendered and updated in real-time according to user actions in the model viewport (Figure 9A, B right-hand side). Once assigned with a flowrate profile, the design tool then generates a print preview to visualize the printed object (Figure $9 \mathrm{C}$ ).

In addition to the layer-by-layer and parallel line infills common in conventional 3D printing, we also implement several new functions to accommodate the generation of non-planar toolpaths. A freeform slicer can take a set of 3D surfaces as input and produce toolpaths by finding their intersections (Figure 9A). Non-planar layers or surfaces can be rasterized with geodesically offset lines to create dense infills (Figure 9B) with equidistant spacing. Lastly, users can also assign toolpaths with non-uniform material flow rates to create variable diameters (Figure 9C). When using the design tool suite, the user can start from either a 3D solid, surface, or polycurve as input and complete the design by assigning flow rates Once complete, a G-code compiler then takes the toolpaths, flow rate assignments, and printing parameters to generate .gcode files

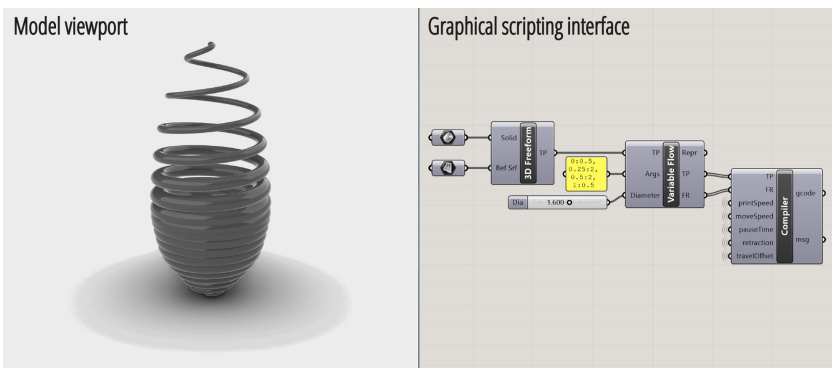

Figure 8: The design tool's interface as seen by the user, which has a model viewport on the left and the graphical scripting interface on the right-hand side.

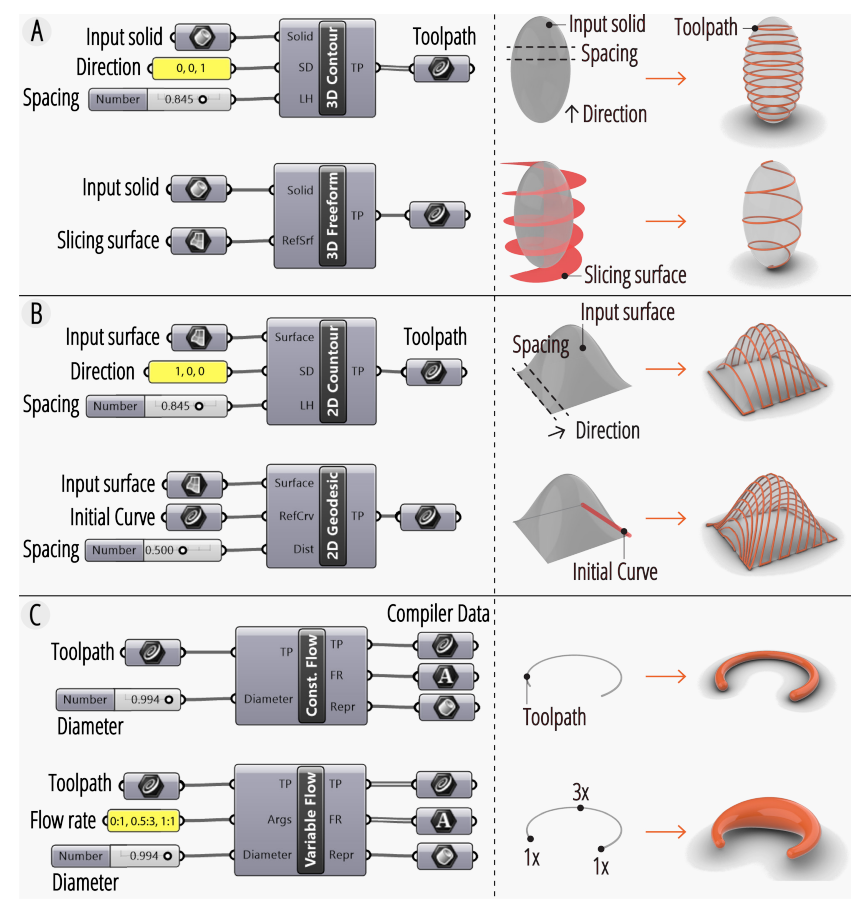

Figure 9: Toolpath design functions - (A) solid to toolpath and $(B)$ surface to toolpath slicing, and (C) toolpath flow rate assignment.

for fabrication. It is worth noting that, because the compiler does not sort the toolpaths nor detect for print path occlusion in any way, the user must manually order the toolpaths before sending them to the compiler.

\subsection{Design Walkthrough}

We take the chocolate jelly art shown in Figure 12B to exemplify the end-to-end fabrication pipeline and design tool workflow using our toolkits. The chocolate syrup has a neutral $\mathrm{pH}$ value and can be printed at room temperature, and the bath is meant to be consumed. We followed the bath selection guideline (Figure 4A) and adopted the curable gelatin bath. Since the bath is non-sacrificial, the release method was omitted. The syrup ink and the gelatin slurry were 


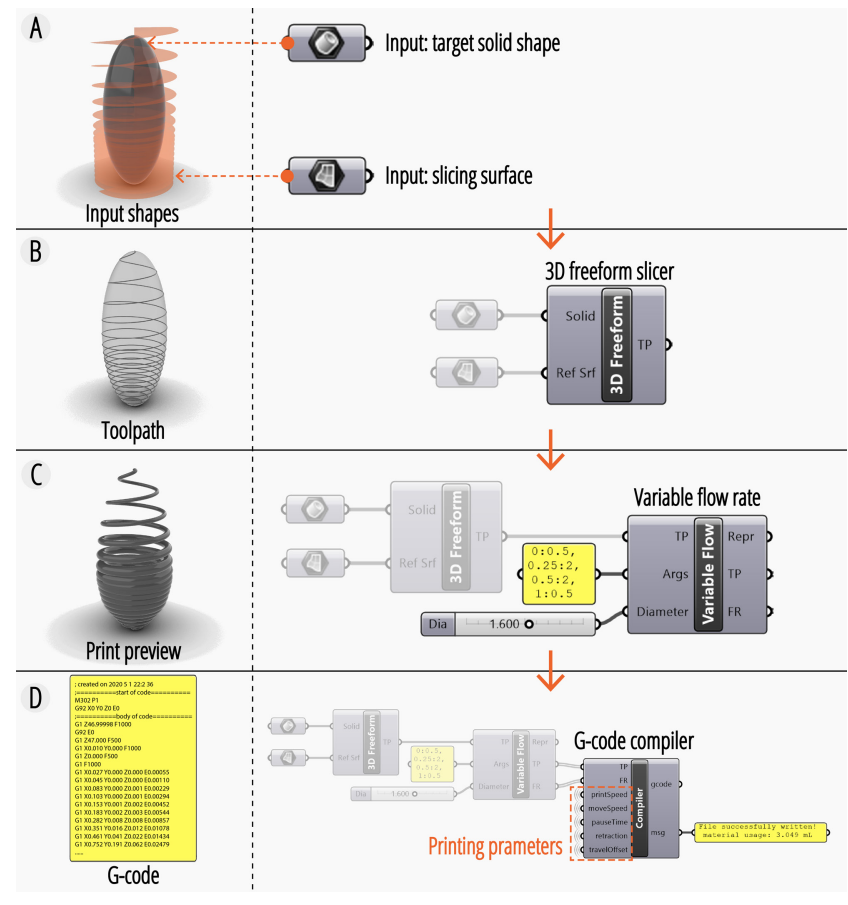

Figure 10: Chocolate jelly art toolpath design walkthrough - (A) modeling input solid and slicing surface as inputs, (B) creating a freeform toolpath using the $3 \mathrm{D}$ freeform slicer, (C) assigning the toolpath with a variable flow rate profile, and (D) generating a .gcode file by specifying the printing parameters.

batch-prepared before the printing session. Since the curable bath has a limited printing window, the gelatin solution was mixed into the slurry right before printing.

To create the .gcode file for this design, we started by modeling the input solid and slicing surface (Figure 10A), then used the 3D freeform solid-to-toolpath function to generate a continuous, non-planar toolpath (Figure 10B). Next, we used the variable flow rate function to assign a decreasing diameter toward the top of the toolpath, and the design tool generated a print preview to visualize the user's design (Figure 10C). Finally, after setting printing parameters, the compiler produced a .gcode file used by our printer (Figure 10D). After printing, the jelly art was placed in a refrigerator for 4 hours to gel thoroughly before consumption.

\section{APPLICATION EXAMPLES}

\subsection{Freeform Chocolate Printing}

In addition to the issue associated with the melted chocolate's fluidity, when printing shapes that are inherently $3 \mathrm{D}$, such as the objects shown in Figure 11A-B, conventional layer-by-layer DIW techniques and slicers would also be unable to capture the smoothness of the model. By contrast, using the Carbopol support bath and our design tools suite (polycurve-to-toolpath function) allows us to create freeform structures (Figure 11C) with ease and broadens the geometrical design space of 3D chocolate printing. Compared

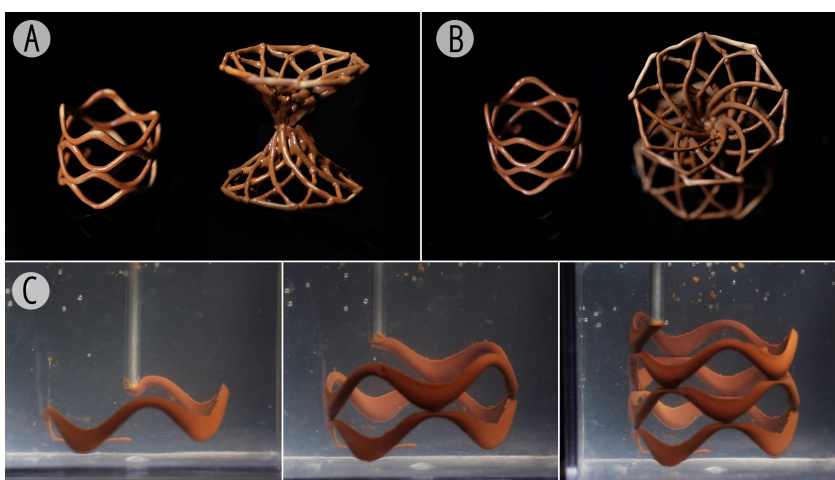

Figure 11: Freeform chocolate art - (A) side and (B) top view, and $(\mathrm{C})$ printing process.

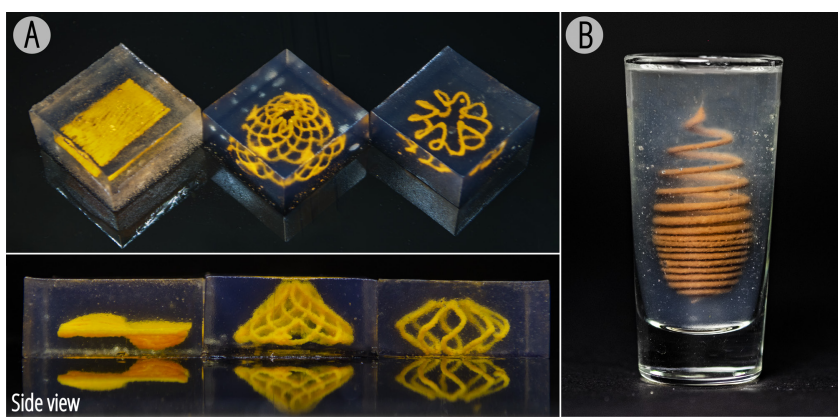

Figure 12: Jelly arts (wagashi) printed with (A) alginatecarrot and (B) chocolate syrup ink.

to [12] that uses a modified chocolate ink (i.e., chocolate syrup + cocoa powder as a rheology modifier), the ink used in this work is an off-the-shelf chocolate bar, thus resembling the texture similar to conventional chocolate treats.

\subsection{Jelly Art}

Wagashi (Japanese-style sweets) uses gelatin as one of the main ingredients, and their making process involves the finesse and craftsmanship of the chef. In this design example, using computationally controlled embedded printing enlarges the shape-design space of wagashi and allows hobbyist makers to design and make personalized dishes in their kitchen. Figure 12 shows several wagashi arts designed and made by the authors - who has no prior experience in making wagashi - in their own houses. The print files were generated using either the geodesic slicing function of the design tool (Figure 12A left) or by manual parametric design in Grasshopper. In addition to the jelly printed with our alginatecarrot ink, Figure 12B also shows a design made of chocolate syrup and printed with a variable printing diameter.

\subsection{Freeform Alginate Printing}

In addition to the jelly art that traps the embedded food inside, we show that the gelatin slurry bath can also be used as a sacrificial bath. The alginate-carrot ink was printed into the slurry bath to create a jellyfish-like design, and the material would be otherwise 


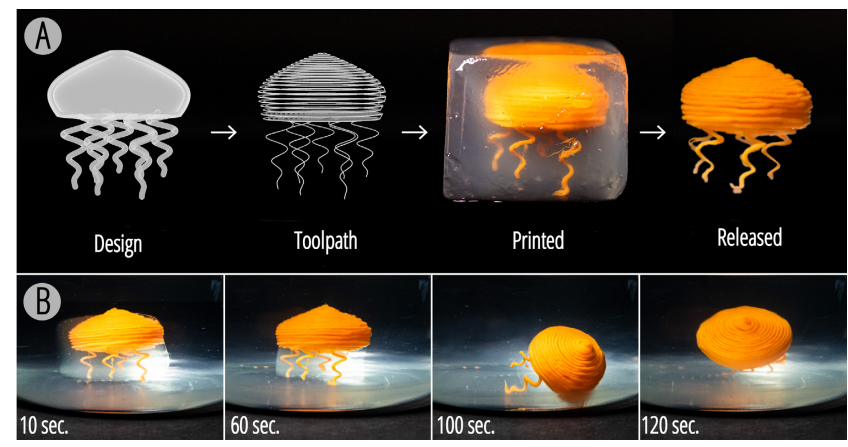

Figure 13: A jellyfish made of the alginate-carrot ink - (A) target shape, printing toolpath, and printed and released jellyfish. (B) The release process showing the gelatin dissolving away in a water bath $\left(60^{\circ} \mathrm{C}\right)$ and the jellyfish becoming adrift.

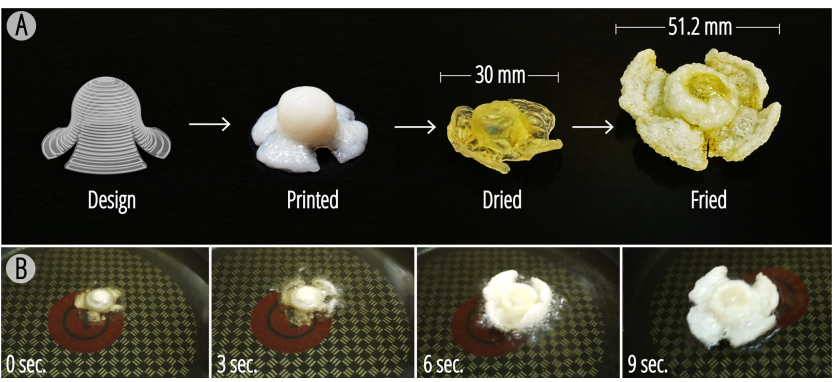

Figure 14: 3D puffable dough - (A) digital models and fabricated objects, and (B) the transformation (frying) process.

difficult to fabricate using DIW due to its inherent softness and fluidity. Figure 13 shows the design, fabrication, and release process of the alginate jellyfish. Once finished printing, the jellyfish and the support bath slurry were removed from the printing container as a whole and submerged into a hot water bath $\left(60^{\circ} \mathrm{C}\right)$ to dissolve away the slurry. Since its density is close to water, the jellyfish could drift inside the water bath with a gentle shake of the container when fully released. Taking this idea further, we believe that similar entrap-and-release designs can also be used for targeted flavor and nutrient release.

\subsection{D Puffable Dough}

Puffing dough in an oil bath is a standard cooking method across cultures. Yet, it is difficult to use DIW to print them into 3D shapes (Figure 8 - 1:1:1.5 column) due to the puffing dough's wetness and fluidity. In this design, by using the Carbopol bath as sacrificial support, we were able to fabricate 3D, flower- and bowl-shaped dough for puffing (Figure 14A, Figure 1E). The toolpath was generated using the layer-by-layer contour slicing function of the design tool, similar to that of a conventional slicer. Anecdotally, we also report that compared to prior $4 \mathrm{D}$ printed food that uses hydration or dehydration for activating transformation [21], fry-puffing can achieve larger transformations ( $70.6 \%$ increase in volume) in a much shorter amount of time ( 9 seconds, Figure 14B).

\section{DISCUSSIONS}

Although our evaluations and design examples show that using support baths can enable freeform and fluidic printing, several material limitations still exist for using our technique. First, neither of the support bath formulations can handle food inks with ionic properties and involve freezing (e.g., ice cream) or boiling during printing and processing steps. Similarly, using hydrogels also requires us to use water-insoluble materials for coloring the printing inks. Future works may consider exploring non-hydrogel-based support bath options to circumvent these limitations. Considering that gelatin is an animal-derived hydrogel for jelly making, it may also be interesting to explore other material options that comply with specific dietary needs, such as vegan-friendly.

In terms of the fabrication platform and design tool, the printer's gantry used in this work has only three degrees of freedom, and the extruder is limited to a vertical orientation. Thus, there are more constraints to consider during toolpath planning (e.g., occlusion, collisions between the needle and the printed material). Although these factors are currently handled by the user, future works may consider developing advanced slicers or algorithms to automate this process. Alternatively, users can also use 6-axis robotic arms for embedded printing [6] and be less constrained by extruder orientation, but doing so would also require an advanced toolpath planner to account for the additional axes. On the other hand, since the support bath retains elasticity under low shear force, some reported that embedded printing is more deformation-prone when printing densely packed lines [1] and produces distorted artifacts. Although we did not find this to be a significant issue in the presented examples and did not occur as a major concern, future works may consider fine-tuning the printing parameters and toolpaths to mitigate this issue.

More than expanding the shape and material design space of food printing, the application examples also show that embedded food printing can enable new human-food interaction modalities, and these examples can be further augmented by future research. For instance, the morphing behavior of puffable dough can be controlled by selectively printing constraint materials (e.g., dietary fibers) to create more complex behaviors (e.g., bending, twisting) and become a $4 \mathrm{D}$ food-printing method. In addition to frying and puffing, it is also possible to bake the steam-cured dough without drying it for making bread. Though not explored in this work, literature [15] also showed that it is possible to control the mouthfeel of food by changing infill patterns, and freeform food printing may provide us with greater customizability of food texture.

Beyond printing edibles, recent HCI literature has also explored methods to functionalize food to become information carriers [21] or displays [10], and similar concepts can be achieved in jellies by using the xanthan gum ink to create a set of hollow, perfusable channels within a gelatin block, such that each channel functions as an individually addressable "pixel" of a display (Figure 15A). Perfusing a channel with dyed water is identical to turning the pixel on (Figure 15B, C), whereas running clear water resembles switching the pixel off. In addition to using jelly as a visual display, we can also produce morphing jelly by perfusing the vasculature with an enzymatic solution to dissolve selected parts, further adding a temporal and shape-changing dimension to edible interfaces. 


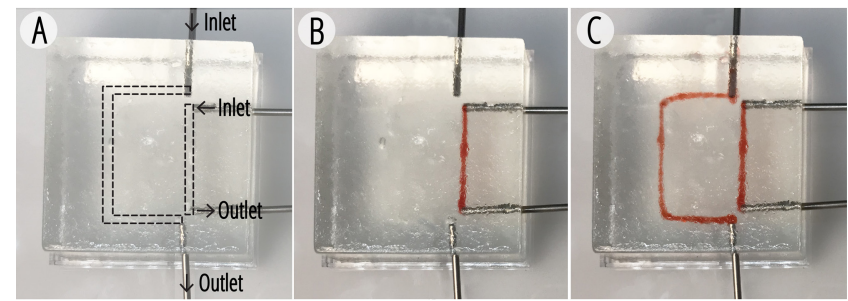

Figure 15: Jelly display - (A) design schematics, and (B) perfusing one segment to show ' 1 ' and (C) both segments to show ' 0 '.

Situated amongst literature, we believe this work expands the design space and narrows the gap between food printing and culinary craftsmanship (e.g., chefs). For either customizing aesthetics or mouthfeels, shapes with large overhangs and wire/mesh structures can be printed with ease. Embedded food printing also accommodates a wider range of material viscosities, which implies that chefs may not need to adjust the recipes, flavor, or texture to achieve printability, making the printed food taste more authentic. We envision that this work will invite a broader audience to adopt food printing and enable HCI researchers to explore human-food interactions in border frontiers.

\section{CONCLUSION}

In this work, we have adapted embedded printing to the fabrication of freeform and fluidic food materials. Several guidelines were presented for readers to select the appropriate support bath type and print-release method depending on the printing material's properties. Our evaluations show that printing into a support bath allows us to produce freeform structures (i.e., large overhangs, bridging, and non-planar toolpaths) with high fidelity and expand the material design space to fluidic food inks, which was otherwise difficult to achieve using conventional 3D food printing methods. A design tool suite was also offered to accommodate freeform slicing and print file generation. Beyond enabling personal fabrication of cuisines, we believe our approach will also enable future HCI works to explore and unlock the interaction potential of morphable and instrumentalized edible interfaces.

\section{ACKNOWLEDGMENTS}

This research was partially supported by the National Science Foundation grant IIS-2017008. We thank Andrew Lee, Neeha Dev Arun, and Adam Feinberg for introducing the embedded printing method, and Angel (Alex) Cabrera, Michael Rivera, Malika Khurana, and the reviewer's comments that helped to improve the quality of this paper.

\section{REFERENCES}

[1] Sara Abdollahi, Alexander Davis, John H. Miller, and Adam W. Feinberg. 2018 Expert-guided optimization for 3D printing of soft and liquid materials. PLoS ONE 13, 4 (2018), e0194890. DOI: https://doi.org/10.1371/journal.pone.0194890

[2] Tapomoy Bhattacharjee, Steven M. Zehnder, Kyle G. Rowe, Suhani Jain, Ryan M. Nixon, W. Gregory Sawyer, and Thomas E. Angelini. 2015. Writing in the granular gel medium. Science Advances 1, 8 (September 2015), e1500655. DOI https://doi.org/10.1126/sciadv.1500655

[3] Thanh Vi Chi, Asier Marzo Pérez, Gianluca Memoli, Emanuela Maggioni, an Damien Ablart. 2020. LeviSense: a platform for the multisensory integration in levitating food and insights into its effect on flavour perception. (2020). DOI: https://doi.org/10.1016/j.ijhcs.2020.102428

[4] chocedge. Choc Edge. Retrieved September 8, 2020 from http://chocedge.com/ home.html

[5] Tom Gayler, Corina Sas, and Vaiva Kalnikaite. 2019. Taste Your Emotions: An Exploration of the Relationship between Taste and Emotional Experience for HCI. In Proceedings of the 2019 on Designing Interactive Systems Conference (DIS '19), Association for Computing Machinery, New York, NY, USA, 1279-1291. DOI: https://doi.org/10.1145/3322276.3322336

[6] Kathleen Hajash, Bjorn Sparrman, Christophe Guberan, Jared Laucks, and Skylar Tibbits. 2017. Large-Scale Rapid Liquid Printing. 3D Printing and Additive Manufacturing 4, 3 (September 2017), 123-132. DOI: https://doi.org/10.1089/3dp. 2017.0037

[7] Evan Hertafeld, Connie Zhang, Zeyuan Jin, Abigail Jakub, Katherine Russell, Yadir Lakehal, Kristina Andreyeva, Sneha Nagaraj Bangalore, Jerson Mezquita, Jonathan Blutinger, and Hod Lipson. 2018. Multi-Material Three-Dimensional Food Printing with Simultaneous Infrared Cooking. 3D Printing and Additive Manufacturing 6, 1 (November 2018), 13-19. DOI: https://doi.org/10.1089/3dp. 2018.0042

[8] Thomas J. Hinton, Andrew Hudson, Kira Pusch, Andrew Lee, and Adam W. Feinberg. 2016. 3D Printing PDMS Elastomer in a Hydrophilic Support Bath via Freeform Reversible Embedding. ACS Biomater. Sci. Eng. 2, 10 (October 2016), 1781-1786. DOI: https://doi.org/10.1021/acsbiomaterials.6b00170

[9] Thomas J. Hinton, Quentin Jallerat, Rachelle N. Palchesko, Joon Hyung Park, Martin S. Grodzicki, Hao-Jan Shue, Mohamed H. Ramadan, Andrew R. Hudson, and Adam W. Feinberg. 2015. Three-dimensional printing of complex biological structures by freeform reversible embedding of suspended hydrogels. Science Advances 1, 9 (October 2015), e1500758. DOI: https://doi.org/10.1126/sciadv.1500758

[10] Ayaka Ishii and Itiro Siio. 2019. BubBowl: Display Vessel Using Electrolysis Bubbles in Drinkable Beverages. In Proceedings of the 32nd Annual ACM Symposium on User Interface Software and Technology (UIST '19), ACM, New Orleans LA USA, 619-623. DOI: https://doi.org/10.1145/3332165.3347923

[11] Viirj Kan, Emma Vargo, Noa Machover, Hiroshi Ishii, Serena Pan, Weixuan Chen, and Yasuaki Kakehi. 2017. Organic Primitives: Synthesis and Design of pHReactive Materials using Molecular I/O for Sensing, Actuation, and Interaction. In Proceedings of the $2017 \mathrm{CHI}$ Conference on Human Factors in Computing Systems (CHI '17), Association for Computing Machinery, New York, NY, USA, 989-1000. DOI: https://doi.org/10.1145/3025453.3025952

[12] Rahul Karyappa and Michinao Hashimoto. 2019. Chocolate-based Ink Threedimensional Printing (Ci3DP). Sci Rep 9, 1 (December 2019), 14178. DOI: https: //doi.org/10.1038/s41598-019-50583-5

[13] Flavius Kehr, Marc Hassenzahl, Matthias Laschke, and Sarah Diefenbach. 2012. A transformational product to improve self-control strength: the chocolate machine. In Proceedings of the SIGCHI Conference on Human Factors in Computing Systems (CHI '12), Association for Computing Machinery, New York, NY, USA, 689-694. DOI: https://doi.org/10.1145/2207676.2207774

[14] Rohit Ashok Khot, Deepti Aggarwal, Ryan Pennings, Larissa Hjorth, and Florian "Floyd" Mueller. 2017. EdiPulse: Investigating a Playful Approach to Selfmonitoring through 3D Printed Chocolate Treats. In Proceedings of the 2017 CHI Conference on Human Factors in Computing Systems (CHI '17), Association for Computing Machinery, New York, NY, USA, 6593-6607. DOI: https: //doi.org/10.1145/3025453.3025980

[15] Ying-Ju Lin, Parinya Punpongsanon, Xin Wen, Daisuke Iwai, Kosuke Sato, Marianna Obrist, and Stefanie Mueller. 2020. FoodFab: Creating Food Perception Illusions using Food 3D Printing. In Proceedings of the $2020 \mathrm{CHI}$ Conference on Human Factors in Computing Systems, ACM, Honolulu HI USA, 1-13. DOI: https://doi.org/10.1145/3313831.3376421

[16] Gayatri Mishra, Dinesh C. Joshi, and B. Kumar Panda. 2014. Popping and puffing of cereal grains: a review. Journal of grain processing and storage 1, 2 (2014), $34-46$.

[17] Moran Mizrahi, Amos Golan, Ariel Bezaleli Mizrahi, Rotem Gruber, Alexander Zoonder Lachnise, and Amit Zoran. 2016. Digital Gastronomy: Methods \& Recipes for Hybrid Cooking. In Proceedings of the 29th Annual Symposium on User Interface Software and Technology, ACM, Tokyo Japan, 541-552. DOI: https: //doi.org/10.1145/2984511.2984528

[18] Marianna Obrist, Yunwen Tu, Lining Yao, and Carlos Velasco. 2019. Space Food Experiences: Designing Passenger's Eating Experiences for Future Space Travel Scenarios. Front. Comput. Sci. 1, (July 2019), 3. DOI: https://doi.org/10.3389/ fcomp.2019.00003

[19] Jeff Potter. 2010. Cooking for Geeks: Real Science, Great Hacks, and Good Food. O'Reilly Media, Inc.

[20] Gabriela Štumberger and Boštjan Vihar. 2018. Freeform Perfusable Microfluidics Embedded in Hydrogel Matrices. Materials 11, 12 (December 2018), 2529. DOI: https://doi.org/10.3390/ma11122529

[21] Ye Tao, Youngwook Do, Humphrey Yang, Yi-Chin Lee, Guanyun Wang, Catherine Mondoa, Jianxun Cui, Wen Wang, and Lining Yao. 2019. Morphlour: Personalized Flour-based Morphing Food Induced by Dehydration or Hydration Method. In Proceedings of the 32nd Annual ACM Symposium on User Interface Software 
and Technology (UIST '19), Association for Computing Machinery, New York, NY, USA, 329-340. DOI: https://doi.org/10.1145/3332165.3347949

[22] Wen Wang, Lining Yao, Teng Zhang, Chin-Yi Cheng, Daniel Levine, and Hirosh Ishii. 2017. Transformative Appetite: Shape-Changing Food Transforms from 2D to 3D by Water Interaction Through Cooking. In Proceedings of the $2017 \mathrm{CHI}$ Conference on Human Factors in Computing Systems (CHI '17), ACM, New York, NY, USA, 6123-6132. DOI: https://doi.org/10.1145/3025453.3026019

[23] Michael Wehner, Ryan L. Truby, Daniel J. Fitzgerald, Bobak Mosadegh, George M. Whitesides, Jennifer A. Lewis, and Robert J. Wood. 2016. An integrated design and fabrication strategy for entirely soft, autonomous robots. Nature 536, 7617 (August 2016), 451-455. DOI: https://doi.org/10.1038/nature19100

[24] Amit Zoran and Dror Cohen. 2018. Digital Konditorei: Programmable Taste Structures using a Modular Mold. In Proceedings of the 2018 CHI Conference on
Human Factors in Computing Systems. Association for Computing Machinery, New York, NY, USA, 1-9. Retrieved September 14, 2020 from https://doi.org/10. $1145 / 3173574.3173974$

[25] 2017. 3D Culinary Printing. 3D Systems. Retrieved August 24, 2020 from https: //www.3dsystems.com/culinary

[26] The Sugar Lab. The Sugar Lab. Retrieved September 16, 2020 from https://www. the-sugar-lab.com

[27] HOME. Nufood. Retrieved January 8, 2021 from http://www.nufood.io

[28] CFR - Code of Federal Regulations Title 21. Retrieved September 13, 2020 from https://www.accessdata.fda.gov/scripts/cdrh/cfdocs/cfcfr/CFRSearch.cfm? fr $=173.73$ 